\title{
Alpha amylase inhibitory activity of some plant extracts with hypoglycemic activity
}

\author{
Rawand S. Abu Soud, Imad I. Hamdan, Fatma U. Afifi* \\ Department of Pharmaceutical Sciences, Faculty of Pharmacy, University of \\ Jordan, Queen Rania Street, Amman, Jordan
}

\begin{abstract}
Thirteen plant species which are claimed to have anti-diabetic activity (based on folk medicine and/or scientific reports) were tested for alpha amylase inhibitory activity. Two of the screened plants exhibited significant (more than $80 \%$ ) alpha amylase inhibitory activity. $I \mathrm{IC}_{50}$ of these plants was estimated based on the dried crude extract and found to be 0.08 , and $0.2 \mathrm{mg} / \mathrm{ml}$ for Aloe vera and Paronychia argentea respectively. In $A$. vera the activity was most likely due to cinnamic acid derivatives. In $P$. argentea the activity was attributed to flavonoid components. These findings support the hypoglycemic activity of these species and give insight about the potential mechanism of their hypoglycemic activity.
\end{abstract}

Keywords: amylase inhibitors, anti-diabetics, Aloe vera, Paronychia argentea.

\section{Introduction}

Several plant species have been advocated in the Jordanian folk medicine for their hypoglycemic effects [1]. The hypoglycemic effect of some of these plants have also been supported by scientific studies on experimental animals [2-7]. However in many cases, neither the active principle nor the mechanism of action have been identified. One possible mechanism for hypoglycemic effect could be through decreasing the absorption of ingested sugars. This could be achieved if the enzyme responsible for the degradation of complex carbohydrates (i.e. alpha amylase) was inhibited. Alpha amylase has been looked at as a potential target to control diabetes since more than 30 years [8]. However it was not before the early nineties when the 
first alpha glucosidase (alpha amylase as well) inhibitor came into clinical use [8]. This inhibitor, known as acarbose, was the result of long screening program for glucosidase inhibitors and has been reported to be quite efficient in controlling blood glucose levels [9]. A major side effect associated with acarbose is flatulence [9]. It has been suggested that alpha amylase inhibitors would result in a better side effect profile (compared to alpha glucosidase inhibitors) as they do not lead to the accumulation of maltose and oligosacharides in the gut [10]. Therefore the aim of this study was to screen some plants commonly used in the folk medicine in Jordan in a search for new alpha amylase inhibitors and to give insight concerning the mechanism of their potential hypoglycemic activity.

\section{Experimental}

\section{Plants and Materials}

All of the examined plants (Table 1) were obtained from the local market (herbalists shops) in a dried form and authenticated by comparison with the herbarium specimens in the Faculty of Science-University of Jordan. A. vera was further characterized according to the identification test described in British Pharmacopoeia, 1993. Emodine, aloin, aloe emodine, barbaloin, hydroquinone and cinnamic acid were obtained from Sigma (USA). Anthracene was from Hnawa Chemicals (China). Purified porcine pancreatic alpha amylase was obtained from ICN Biochemicals (Ohio, USA). All other reagents and solvents were obtained from Merck (Germany). Distilled water was used for the preparation of the different solutions.

\section{Preparation of the extracts}

All plants were extracted with $50 \%$ methanol in water according to the method described by Hansawasdi [11] with some modifications. The dried plant was ground to pass through a 40-mesh sieve. Of the sieved material $1 \mathrm{~g}$ was extracted with $10 \mathrm{ml}$ of $50 \%$ methanol water by incubating in a water bath at $50{ }^{\circ} \mathrm{C}$ for 30 minutes. The obtained extract was centrifuged and filtered before being subjected to alpha amylase inhibitory test. 
For the purpose of estimating the $I_{50}$, the concentration of the whole extract was estimated by evaporating the solvent to dryness (using rotary evaporator) and weighing the dried residue. The percentage yields (based on the dried residue) together with the result of the phytochemical screening, according to Wagner [12] for all of the examined plant extracts are presented in Table 1.

\section{Alpha amylase inhibitory test}

lodine solution was prepared by dissolving $0.254 \mathrm{~g} \mathrm{I}_{2}$ and $4.0 \mathrm{~g} \mathrm{KI}$ in $1 \mathrm{~L}$ of distilled water. Starch solution was prepared by dissolving $1 \mathrm{~g}$ of starch in $10 \mathrm{ml}$ of distilled water, gently boiling, cooling and completing to $100 \mathrm{ml}$ with distilled water. Amylase solution was prepared by transferring $6 \mu$ of the standard porcine pancreatic amylase suspension $(40 \mathrm{mg} / \mathrm{ml})$ to $8 \mathrm{ml}$ of phosphate buffer $(\mathrm{pH} 6.9)$.

Alpha amylase inhibitory activity was based on the starch-iodine method described by Hansawasdi [11] with some modifications. Briefly, control and test solutions were prepared as follows: $0.3 \mathrm{ml}$ of amylase solution were transferred to a sample tube containing $0.3 \mathrm{ml}$ of the extract to be tested (substituted by the solvent of extraction in the case of control) and $0.6 \mathrm{ml}$ phosphate buffer $(\mathrm{pH} \mathrm{6.9)}$. The mixture was incubated at $37{ }^{\circ} \mathrm{C}$ for 15 minutes. $0.4 \mathrm{ml}$ aliquots of that incubate were transferred to sample tubes containing $3 \mathrm{ml}$ starch $(1 \mathrm{~g} \%)$ and $2 \mathrm{ml}$ of phosphate buffer (pH 6.5) and the mixture was re-incubated for 45 minutes. At zero time and at the end of the incubation period $0.1 \mathrm{ml}$ of the reaction mixture was withdrawn from each tube after mixing and discharged into $10 \mathrm{ml}$ of iodine solution. Solutions were thoroughly mixed and the absorbance measured immediately at $565 \mathrm{~nm}$. Percentage inhibition was calculated according to the formula:

$\left[\left(A_{0}-A_{t}\right)\right.$ control $-\left(A_{0}-A_{t}\right)$ sample $\left./\left(A_{0}-A_{t}\right)_{\text {control }}\right] \times 100 \%$

where $A_{0}$ and $A_{t}$ are the absorbance values at zero time and at the end of the incubation, respectively. Each experiment was repeated three to four times and the average value was used for obtaining the relevant plots. 


\section{Results and discussion}

Thirteen plant extracts were tested for alpha amylase inhibitory activity using a literature method that was optimized and validated in the author's labs [13]. Results were calculated as percentage inhibition (under experimental conditions) and summarized in Table 1. Of the thirteen plant species tested only two extracts exhibited significant (more than $80 \%$ ) alpha amylase inhibitory activity; namely $A$. vera, and $P$. argentea. For these plants the inhibitory activity was also shown to be concentration dependent. Dose response curves were obtained for the two most active plant extracts and compared to that of acarbose as a standard alpha glucosidase and amylase inhibitor (Fig. 1). The $I C_{50}$ of each plant extract was determined (from Fig. 1) as the inhibitor concentration (estimated on basis of the dried extract) required to inhibit $50 \%$ of alpha amylase activity under the described experimental conditions. Acarbose appeared to be about 20 times more active $\left(I C_{50}=0.004+1-0.0003 \mathrm{mg} / \mathrm{ml}\right)$ than $A$. vera $\left(\mathrm{IC}_{50}=0.081+/-0.003 \mathrm{mg} / \mathrm{ml}\right)$ and 50 times more active than $P$. argentea $\left(\mathrm{IC}_{50}=\right.$ $0.20+/-0.02 \mathrm{mg} / \mathrm{ml}$ ). However, the two plant extracts are considered to have a significant inhibitory activity that might contribute to their claimed anti-diabetic activities. Particularly because the $\mathrm{IC}_{50}$ was estimated based on the weight of the crude dried extract in this experiment, and thus has to be higher than the $\mathrm{IC}_{50}$ of the respective pure active component responsible for the effect.

Although both of these plants have been advocated in folk medicine for antidiabetic activity [1] only $A$. vera has been shown by scientific studies to have such activity [14]. Therefore alpha amylase inhibition is responsible (at least in part) to the hypoglycemic activity of $A$. vera, and $P$. argentea. As regard to other plants which did not show significant alpha amylase inhibitory activity they may still be active as hypoglycemic plants but through a different mechanism of action; particularly those which have been supported by scientific studies on animals. 


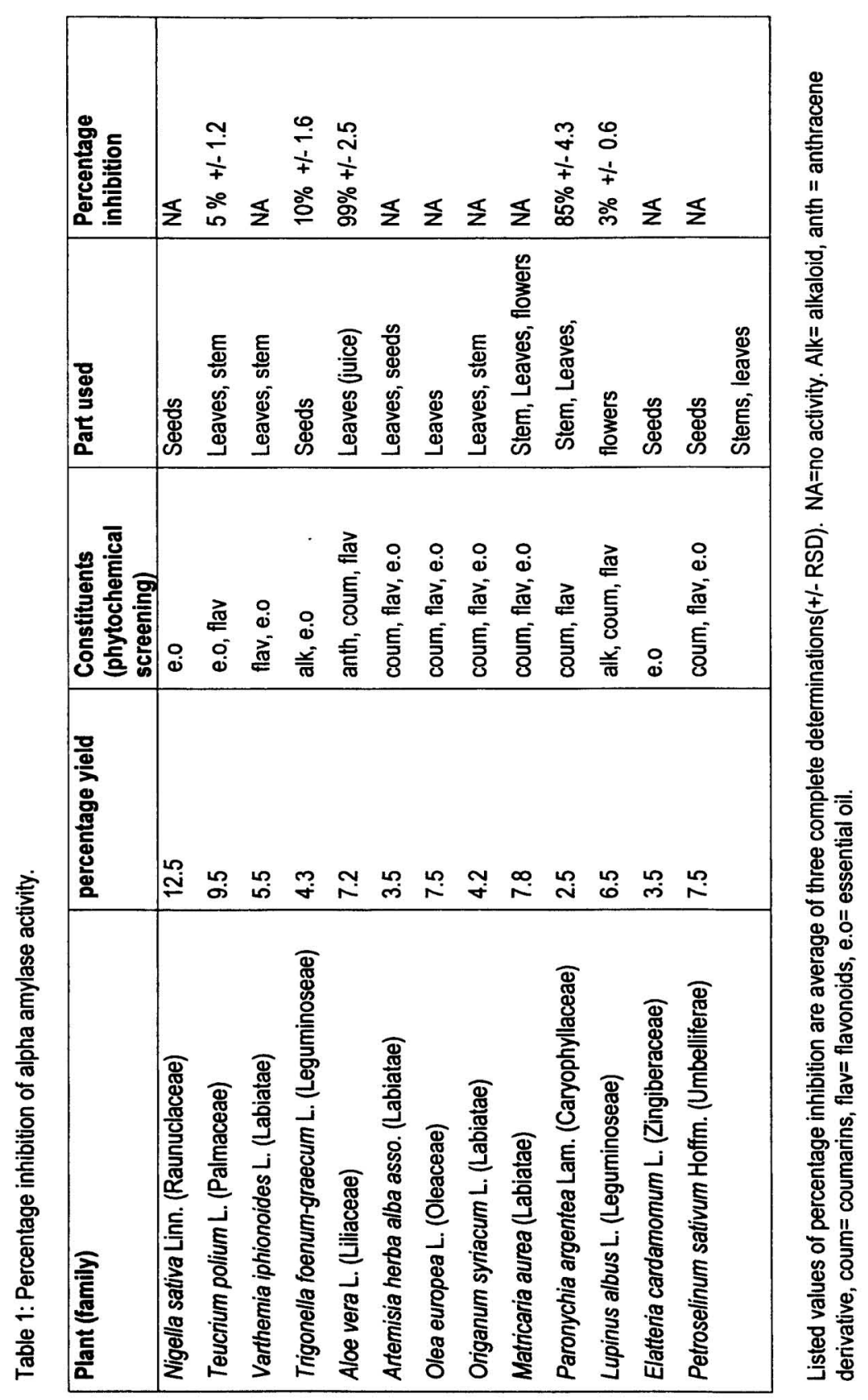




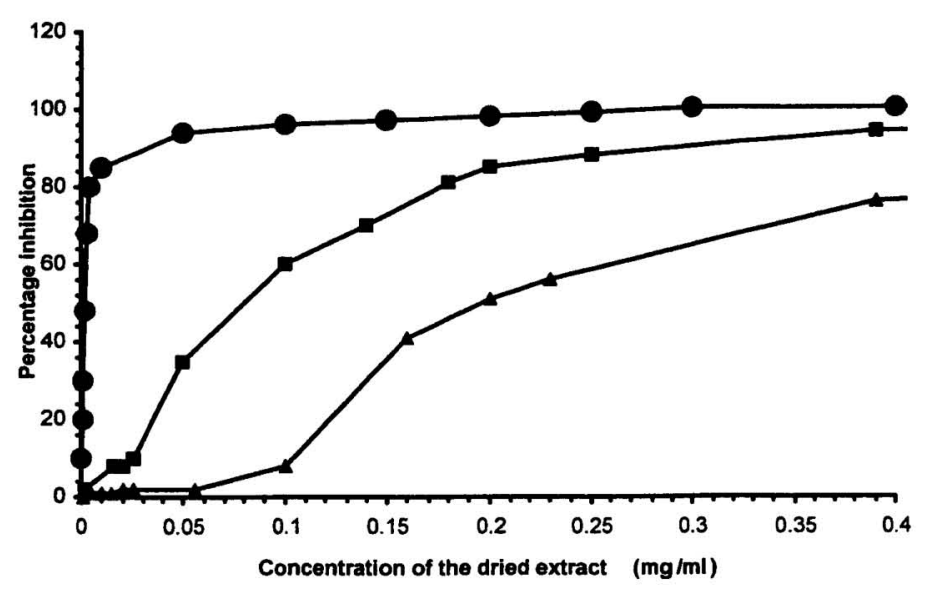

Fig. 1: Dose response curves for acarbose $(\bullet)$ and the crude extracts of $A$. vera $(\square)$ and $P$. argentea $(\boldsymbol{\Delta})$. At each concentration point the average of three independent measurements was used for constructing the plot. R.S.D. was less than 5 in all cases.

Since $A$. vera is one of the well studied plants with known-major constituents, attempts to identify the biologically active principles were made by testing the commercially available purified constituents. Major constituents of $A$. vera include the anthraquinone derivatives: emodine, aloin and aloe emodine. When tested for alpha amylase inhibitory activity emodine and aloe-emodine exhibited maximum of 1.5 and $2.5 \%$ inhibition at saturated concentrations respectively, but aloin showed no activity up to concentrations of $20 \mathrm{mg} / \mathrm{ml}$. It was thought that the observed low activity was attributed to the low solubility of these compounds in the test solvent (methanol:water).

It might be possible that other constituents of the whole extract would facilitate the solubility of anthraquinones leading to higher activity. Addition of surfactant (tween 80 ) to the test solvent (and the control) was found to improve the solubility of both of emodine and aloe-emodine. The observed increase in solubility was accompanied by increase in activity: $9 \%$ and $16 \%$ for emodine and aloemodine, respectively. Thus the 
activity of the purified emodine and aloemodine was still far below that observed for the whole extract $(100 \%)$ which suggested the presence of other active constituents. Anthracene, anthracene-9-carboxylic acid, anthraquinone, 1,2,5,8 tetrahydroxyanthraquinone, and hydroquinone are other related anthraquinone derivatives that were available in pure form. When these were tested for alpha amylase inhibitory activity no inhibition was observed even at saturation concentrations. Therefore, the high alpha amylase inhibitory activity could not be attributed to the anthraquinone derivatives.

Other reported constituents of $\boldsymbol{A}$. vera include ester derivatives of parahydroxy cinnamic acid [12]. As these were not available, pure cinnamic acid and cinnamaldehyde were tested using the same procedure. At saturation concentrations a maximum of $30 \%$ inhibition was observed for the pure cinnamic acid and zero inhibition for cinnamaldehyde. In order to ensure that the effect of cinnamic acid was a specific one rather than a non specific deactivation by decreasing the $\mathrm{pH}$ of the medium, the $\mathrm{pH}$ was measured and found to be within the expected range (6.6). These findings tend to suggest that the principal activity resides in one or more of the hydroxy cinnamic acid derivatives. For further evidence, the extract was run on preparative TLC plates using a standard mobile phase that is recommended for the separation of the constituents of A. vera [ 12]. Aloin and aloe-emodine were used as standards on a separate lane on the TLC plate. After development and visualization under UV light spots were removed from the plate and subjected to alpha amylase inhibition test.

The obtained TLC-chromatograms were comparable to the reference one and showed 14 distinguishable spots. According to Wagner et al. [12] the spots above aloin and below aloe-emodine (obtained $R_{f} 0.5$-.99) were attributed to various cinnamic acid derivatives e.g. p-coumaric acid methyl ester which exhibit blue fluorescence under the UV light $365 \mathrm{~nm}$. Of the fourteen fractions examined only two exhibited significant activity; those were with $R_{f}$ values of 0.91 and 0.61 and produced inhibitory activity of $37+/-4 \%$ and $30+/-2 \%$ respectively. When examined under UV light at $365 \mathrm{~nm}$, these fractions exhibited blue fluorescence. Thus combining these data with those obtained from experiments on purified constituents it would be evident that the alpha amylase 
inhibitory activity of $A$. vera resides in some of the cinnamic acid derivatives rather than the anthraquinone derivatives.

It would be expected that amylase inhibitors should possess a sugar-like structure e.g. acarbose. However alpha amylase inhibitory activity has been demonstrated for other non-sugar naturally occurring products such as hibiscus acid [11] and flavonoids including luteolin. As the carboxyl group is the common structural feature between cinnamic acid and hibiscus acid it might be possible that carboxylic acid group together with other structural features (e.g. size) is important for alpha amylase inhibitory activity. However, further studies are needed and currently in progress to isolate and identify the constituents responsible for alpha amylase inhibitory activity in the active plant species $A$. vera, and $P$. argentea.

\section{Acknowledgement}

The authors thank Abdul Hameed Shoman Foundation for their financial support throughout the project.

\section{References}

1. Abu Irmailah B, Afifi F. Treatment with medicinal plants in Jordan.

Dirasat Biological and Medical Sciences 2000; 27:53-74.

2. Ajabnoor M, Tilmisany A J.

Effect of Trigonella foenum graecum on blood glucose levels in normal and alloxan diabetic mice.

J. Ethnopharmacol. 1988; 22: 45-49.

3. Gharaibeh M, Elyan H, Salhab A J.

Hypoglycemic effects of Teucrium polium.

J. Ethnopharmacol. 1988; 24: 93-99.

4. Ribes G, Sauvaire Y, DaCosta C.

Antidiabetic effects of subfractions from fenugreek seeds in diabetic dogs.

Proc. Soc. Exp. Biol. Med 1986;182: 159-1-166.

5. Sharma R, Raghuram $T$, Rao $N$.

Effect of fenugreek seeds on blood glucose and serum lipids in type 1 diabeties.

Eur. J. Clin. Nutr.1990; 44: 301-306.

6. Al-Rahman A, Zaman K J. 
Medicinal plants with hypoglycemic activity.

J. Ethnopharmacol. 1989; 26: 1-55.

7. Afifi $F$.

Int. J. Pharmacog. 1993; 31: 161.

8. Jung B, Matzke $M$, Stoltefus J, editors.

Chemistry and structure activity relationships of glucosidase inhibitors.

In: Handbook of experimental pharmacology: Oral antidiabetics.

Berlin: Springer-Verlag, 1996: 411-425.

9. Lebovitz $\mathrm{H}$.

Alpha glucosidase inhibitors as agents in the treatment of diabetes.

Diabetes Reviews 1998; 6:132-145.

10. Kim J, Kown C, Song K.

Inhibition of alpha-glucosidase and amylase by luteolin, a flavonoid.

Biosci. Biotech. Biochem. 2000; 64: 2458-2461.

11. Hansawasdi C, Kawabata J, Kasai T.

Alpha amylase inhibitors from Roselle (Hibiscus sabdariffa Linn.) tea.

Biosc. Biotech. Biochem. 2000; 64:1041-1043.

12. Wagner $\mathrm{H}$, Bladt $\mathrm{S}$.

Plant drug analysis; $A$ thin layer chromatography atlas, 2nd Edn,

Berlin: Springer-Verlag, 1984.

13. Abu Soud R, Afifi F, Hamdan I, MSc. Thesis, Department of Pharmaceutical Sciences, University of Jordan, 2002.

13. Okyar A, Can A, Akev N, Baktir G, Sutlupinar N.

Effect of Aloe Vera leaves on blood glucose level in type I and type II diabetic rat models.

Phytotherapy Res. 2001;15: 157-161. 